\title{
Forty Years' Study of Color
}

\author{
By Katsuhiro Yamasaki, Member, TMSJ \\ Nara Women's University, Nara
}

Based on Journal of the Textile Machinery Society of Japan, 18, 250-256 (1964)

\begin{abstract}
No study of color effect is complete unless it deals with at least three aspects: physics, physiology and psychology. This article covers only a phase of the author's 40year study of color effect, but it goes into a problem not covered in the Universal Book of Color Science. The highlights of this article are :

1) The author has devised a formula for producing multiple colors out of two with a special instrument called Muto Chrome.

2) He has been able to establish physiologically that a colorless state looks colored.

3) He has completed a quantative analysis of a complementary after-image.

4) He has devised a method of more exact and more rapid color-matching.
\end{abstract}

\section{Introduction}

Roughly speaking, color has to be studied from three major points of view: physical, physiological and psychological. A scientific study of color from any one of these viewpoints will take longer than a lifetime. A practical study of the problem of color is incomplete if we ignore any one of the three viewpoints.

Since color is visible to the naked eye, we need, first of all, a knowledge of the work the eyes do. In order that the eyes may do their work, there must be a reflection or transmission of color light from the outside. Also we must interpret an image of light or color transmitted from the eyes to the center of the brain.

Only after we have passed physical, physiological and psychological processes can we claim to have seen color.

It often happens that the same color that we observe under identical physical condition looks different. To find out why, I began experiments. That was the beginning of my study of color.

I tried to catch the certain phenomenon of color effect quantitatively, but the task proved more difficult than I had thought, because I was faced with a phenomenon related to sensation and perception.

It is possible, however, to draw abstract and conceptual conclusions on a phenomenon related to sensation and perception from the sum total of the uninfluenced opinions, or conclusions, of people experimented on. To examine unsolved problems about color effect analytically, we must, at least, take the sum total of the uninfluenced opinions of people experimented on. In fact, by doing so, I have been able to solve a number of problems in the last 40 years.
One problem remains unsolved: How to express abstract and conceptual conclusions quantitatively. I may be unable to solve the problem during the rest of my life. However, science is to be corrected, supplemented and improved by succeeding generations. I write this article hoping that some future scientist will solve the problem if I cannot in my lifetime.

\section{Muto Chrome}

A machine made in England, called Muto Chrome and used in my laboratory can freely change the color effect of the ground and pattern of the design of dyeing and weaving. The machine is, therefore, handy for improving the efficiency of color design. The machine changes color effect by controlling the irises of seven lenses and by using a large number of colored filters. Colorful color effects thus obtained are excellent beyond expectation.

I have lived with Muto Chrome for many years. One day someone called me up by phone while I was making an experiment in a dark room. I rushed out to answer the phone. When I returned, I found that the color effect reflected on the screen was quite different from what I had looked at a little while before. I found a rough color tone and a soft color I had never seen before. I had gone in and out of the room countless times before, but I had never seen color effect change so suddenly. I looked in an ecstacy of admiration at the change.

I then walked over to Muto Chrome, intending to make the next experiment, and had another pleasant surprise. There should have been three color filters of 
red, green and blue, but now the blue filter was gone and only white light was transmitted from where the blue light should be. Only the red and green filters remained. I set my eyes upon the screen again and saw the beautiful color effect there all the same.

Until then I believed firmly in the old theory that at least three primary colors were needed to produce multi colors. Now I was seized with the "pleasure of exploration" such as Columbus must have felt when he discovered the American continent. I began to explore the unknown. For 40 years since then, I have grappled with the same problem day in, day out, often being ridiculed as "color crazy." I have had to make all machine parts necessary for my experiments myself. All because I was enchanted with Muto Chrome.

\section{Experiment to Change Non-Color}

\section{(Colorlessness) to Color}

Muto Chrome has seven lenses. Use only two which stand horizontally. Uncap them and put a red filter on one of them. Leave the other as it is, adjusting only its irises. Make the lights on the left and right equal in lux. Put them into full contact on the screen. Prop up a stick $3 \mathrm{~cm}$ in diameter exactly bet. ween the light source and the screen. The stick then casts two projections on the screen.

All this is common knowledge, but no spectators can correctly give off hand the colors of the projections. Some may give them as red and white; the captious, as red and grey.

Table 1. Relation Between Color of Light Source and Projected Color

\begin{tabular}{c|ccccc}
\hline $\begin{array}{c}\text { Color of } \\
\text { light } \\
\text { source }\end{array}$ & Red & Orange & Yellow & Green & Purple \\
\cline { 2 - 6 } & White & White & White & White & White \\
\hline $\begin{array}{c}\text { Projected } \\
\text { color of } \\
\text { a stick }\end{array}$ & Red & Oange & Yellow & Green & Purple \\
green & Blue & $\begin{array}{c}\text { Purple } \\
\text { blue }\end{array}$ & $\begin{array}{c}\text { Red } \\
\text { purple }\end{array}$ & $\begin{array}{c}\text { Yellow } \\
\text { green }\end{array}$ \\
$\begin{array}{c}\text { Color of } \\
\text { background }\end{array}$ & Pink & $\begin{array}{c}\text { Salmon } \\
\text { pink }\end{array}$ & Cream & $\begin{array}{c}\text { Pale } \\
\text { green }\end{array}$ & $\begin{array}{c}\text { Pale } \\
\text { purple }\end{array}$ \\
\hline
\end{tabular}

In my experience, all spectators, even though told beforehand about the projections, expressed amazement at what they saw - except on one occasion. A young man, looking quite uninterested, asked, much to my surprise: "What in the world are you doing this experiment for?" I learned later that he always painted only in white, black, grey, darkbrown and greyish green, that his paintings were really bold yet calm in color tone and that his skill in design was superb. In short, he was a color-blind painter.

This experience started in me the idea of using the same experiment for examining color blindness. With this idea in mind, I visited a laboratory of ophthalmology, but was told an "inspected vote of the color blind" - results of surveys of the color blind - by Dr. Shinobu Ishihara was universally used and precluded the use of an experiment made by a layman like myself. I then decided I would make it my life work to try and find out why non-color changes to color.

\section{3-1. Why Does Projected Color Which should Be Non-color Look Colored?}

I found a little later that the key to this problem was related to the movement of the eyes. I visited laboratories of ophthalmology and otorhinolaryngology to delve into the theory of boundary contrast propounded by M. E. Chevreul. I learned that the laboratory people had come out with an experimental theory on aeronau tical medicine. The theory was that the movement of the eyes is induced by the movement of an external body. I was one of the people on whom they made experiments to put their theory to the test, and I some how agreed with the theory.

One of the experiments was made in this way: I entered a pipeshaped cylinder as large as $2 \mathrm{~m}$ in diameter hanging from the ceiling. I found inside the cylinder red stripes on a white ground pasted perpen dicularly at intervals of $30 \mathrm{~cm}$. On a signal from me the pipe began to be turned quietly. I received the gymnastic command: "Mark time." I did the stepping. After about three minutes of this, the cylinder stopped turning and I found myself in the opposite direction. I then stepped out. I was told: "You've seen for yourself, while your eyes were chasing the red stripes on the white ground, your feet shifted by stepping."

"Yes, indeed, thank you," I answered, but I found myself still far from the answer to my problem, I pon dered and pondered, then it dawned on me that, by undergoing the experiment just mentioned, I had figuratively speaking - tried to weigh a pearl with scales with which to weigh an elephant.

One day I had an opportunity to read an article entitled "Measuring the Movement of the Eyeballs during Staring" by Yasuo Okuse in the Journal of the Ophthalmic Institution of Japan. I found it a detailed and thorough report on an experiment. The article made me aware that, while we thought we were staring at one point, our eyes always moved up and down and to right and left about $20^{\prime}$ in visual angle. I began trying to find out why the width of the movement of the eyes was almost a regular quantity, i.e., about $20^{\prime}$ 
in visual angle. I was not entirely without a clue, because I had had previous knowledge of the relation between the excitement and stimulation of the nerves.

S. L. Polyak, M. D., says, in effect, in "The Retina" (1948): "Two thousand cones mass within about 100 microns in diameter and about $20^{\prime}$ in visual angle of the central fovea of Retina." I found from many experiments that the sensibility of the cone was highest about $1 / 2$ sec. after we began watching color, declined to about $50 \%$ after about $1 \mathrm{sec}$. but never declined further and kept itself constant.

When the cone which receives color stimulus first declines in sensitivity another cone replaces it and tries to increase its sensitivity. Presumably, such a physilogical phenomenon induces a tremor of the eyeballs.

The next problem is that the width of the area in which cones mass and the width of the movement of the eyeballs are both about $20^{\prime}$ in visual angle. In short, the boundary contrast M. E. Chevereul speaks of is a phenomenon which occurs when we stare at the boundary of color and color. The movement of the eyeballs at that time is about $20^{\prime}$ in visual angle. Cones reach the highest sensibility $1 / 2 \mathrm{sec}$. after receiving color stimulus and declines in sensitivity to about $50 \%$ after 1 sec., thus inevitably setting off the process of cones replacing one another. This replacement takes place only among 2, 000 cones which form the central fovea. Presumably, then, the movement of the eyeballs occurring with such a process is limited within $20^{\prime}$ in visual angle. These factors enabled me to solve the difficult problem of why non-color changes to color.

\section{Meaning of Complementary After-Image}

I have just about completed my explanation of why a non-color area changes to color, but another important problem awaits solution. We have noted that the cone is the highest in sensibility $1 / 2$ sec. after we begin watching a color, declines to $50 \%$ after $1 \mathrm{sec}$. and never declines further. The question, then, arises: What is a decline in color sense?

Looking at red cloth, we cannot tell how much the color has actually changed. Arranging two pieces of colored cloth, one red and one green, side by side and staring at the boundary, we at once find a bright, purplish red area on the red side of the boundary and a bright, bluish green area on the green side alternately. If we watch left and right closely at this time, we find what seems to be noticeable fading of color. The inference is deducible that there is a difference of about $50 \%$ in chroma.

The boundary contrast of red and green shows up in bluish green produced presumably by blue green (complementary after-image of red) coming on top of neighboring green; and shows up in purple red produced presumably by red purple coming on top of neighboring red. In the other parts, however, blue green (complementary after-image of red) comes on top of red, while red purple (after-image of green) comes on top of green.

However, (1) red and blue green and (2) green and red purple are complementary to each other and should theoretically become non-color if combined. Actually, since a complementary after-image is weaker than a real image, (1) and (2), combined, do not become non-color, but become close to non-color, red turning muddy red and green muddy green.

\section{Quantitative Analysis of Complementary}

\section{After-Image}

Any one who has made repeated experiments on boundary contrast is aware, I believe, that the difference between the vividness of the boundary and the rough. ness of the other areas exceeds prediction. It often hap. pens also that, in selecting a color for painting, dyeing or for a cloth, we pick up only agnate colors, make a choice after much debating - and then want to correct our choice. This has been taken as a matter of course in the world of color.

Architectural superintendents, painters and people engaged in color-watching have, doubtless, been seeking a way out of such hazards. I have been one of them. Not only that, I have been trying night and day to devise a way out.

While I was looking at a color vote during an experiment on after-image, I found colors equal to the colors of the vote protruding from above, below and left and right of the grey mount. They were vivid. I was struck with an idea: They were not complementary colors but were to be explained thus: One second after I began looking at a color, the complementary after-image of the color vote showed up, went on top of the vote and shifted in whatever direction in which the eyes shifted.

In traditional experiments on after-image, a small piece of colored paper is placed on white paper, beheld for a brief spell and removed rapidly. Then colors equal to the complementary colors of the vote show up where the piece of colored paper was.

Reports of the traditional experiments have been literally translated and are found in all books on color science. Some books contain blank pages marked with a circle or an $X$ in the center, obviously intended to rivet the reader's eyes to the mark.

My method is to keep a complementary after-image within the outline by drawing an outline which is the same in shape and size as the color vote. It is very 
difficult to watch complementary after-image distinctly by other methods without skill.

I made a measurement with a special apparatus to find out how much of a complementary after-image showed up. The method was to generate a complementary after-image, let it vanish and take the ratio of the quantity of color used to let the complementary afterimage vanish to the amount of the test color vote as a complimentary after-image.

Make the following assumptions:

Given color vote …......... F

Color degree $1 \mathrm{sec}$. after we begin staring at it ............. $f_{a}$

Color degree of complementary after-image

Grey mask equal in value to $F \quad \ldots \ldots \ldots \ldots . . . N$

On these assumptions, $N$, under the influence of $F$, shows up in $f_{b}$, which is the color of the complementary after image. Besides, $f_{a}$ and $f_{b}$ being complementary colors to each other, are $f_{a}+f_{b}=N$. Therefore $N$ $1 \mathrm{sec}$ after receiving the influence of $F$ is in the relation of $N-f_{a}=f_{b}$. Therefore, to restore $N-f_{a}$ to the original $N$, we must make it $N=f_{b}+f_{a}$.

In short, we must add the same color as the test color vote to grey to make the grey look the original grey while the grey mask equal in value to the test color vote is covered with the complementary after-image of the test color vote. At this time, the amount of color used to let the after-image vanish equals the complementary after-image. I was able, by this experiment, to prove that chroma declined about 50\% if we looked at the same color for a long spell.

Let me repeat: If we look at a color, color sense equal to the complementary colors of that color is sure to arise and it is piled up on the color, resulting in a decline of about $50 \%$ in cbroma. If two colors line up which are complementary to each other, their comple. mentary after-images are piled up on top of each other with the movement of the eyes. The result is that the color which is the same as the complementary colors increases in brilliance.

On the contrary, if light and dark agnate colors, e.g., red and pink, line up, then blue green (the complementary color of red) is piled up on the pink, making the pink more like grey. This is worthy of special mention as a case where a color does not change in hue by combination with another. In all other cases, the original color changes in hue by being piled up with the complementary after-image of another color with which the original is combined. If color is difficult to handle, it is, I think, because we do not know enough about the influence of complementary after-image.

\section{Knack of Color-Matching}

The problem of color-matching is always taken seriously, but we have no cut and dried knack of color. matching. If a color matches another at all, it is ac. cepted as satisfactory. Most experts have no alternative but to depend on their intuition which they have culti. vated over the years. When I tried color-matching myself, however, I found it nothing difficult. My long, laborious previous study of complementary after-image helped me far more than I had expected.

The method generally used to see if colors $\mathrm{A}$ and $B$ are equal is to put them side by side and compare them by observation. An objection to this method is that even the same color of ten looks different unless we use a flat test piece. The reason is that, when two colored test pieces are arranged side by side, the influ ence of the boundary contrast is apt to emphasize even a slight error in the boundary.

Long ago our forefathers learned to measure color. The way we use of placing two colors side by side for comparison was their first discovery. It is one of the most primitive ways, but the use of a spectro photo. meter entails heavy expenses and requires a lot of time for after-adjustment. A conceivable alternative is to measure two colors separately with a fixed rule in search of equality between them. A similar way has to be devised for comparing colors also.

A "fixed rule" means a grey mask having two open windows. By letting a standard color vote peep out of one window and a test color vote out of the other window and comparing the relation between grey and the standard color vote and the relation between grey and the test color vote, we can easily decide whether A and B match each other. We call this method colormatching by separating.

Once, when I made an experiment, I debated within myself for a long time as to which grey to select. I then used a wide variety of grey masks and asked the people on whom I was experimenting to specify parti. cular greys they found easy to compare. $N 7 /, N 6.5 /$ and $N 6 /$ of Munsell color votes won many ballots; N5/, $N 9.5$ / and N1.5/, only a few. By consensus, N6.5/土 0.5 was a proper range. There still were problems awaiting solution, however.

I found it necessary to put in black outlines, or divided lines, on the edge of the window through which colors varying in the degree of brightness - high, medium and low - could peep out and be seen clearly.

I covered this point in an article entitled "Effects and Results of Divided Lines in Color Harmony" in the Journal of Home Economics, Vol. 1, No. 3 (1952). I 
omit discussion of it here. Suffice it to say that the effects of divided lines showed up clearly in a grey mask and proved useful for promoting the efficiency of color-matching. It seems, however, that this method is hardly used even today. The grey mask attached to the "OBC Textile Color Manual Color Chart" published by Oversea Books Co., Inc. is the first example of the use of this method.

This grey mask is worked out by calculating the width of windows and the space between windows on the basis of a $50-\mathrm{cm}$ visual distance. A study on how to fix the window width was published in my article "Relation between Area of Color Vote and Distance of Observation" in the Journal in Home Economics, Vol. 7 , No. 2 (1956). The gist of the article is that $R$ (ratio) of $L$ (visual distance) and $W$ (width of color vote) make the following relation:

$$
R=\frac{W}{L}=0.04
$$

Therefore, with a $50-\mathrm{cm}$ visual distance the formula (1) is : $\quad W=R L=2 \mathrm{~cm}$

Assuming that a divided line is $w$ in width, the formula is :

$$
w \leqq 1 / 20 W
$$

Assuming the inter-window space to be $W^{\prime}$, the formula is :

$$
W^{\prime} \geqq W
$$

A mask varies, though slightly, in size depending on the use to which it is put but needs a space more than the combined total of the length and breadth of the windows around the windows. To use a mask having this space is to apply the law of successive contrasts. Therefore, we must consciously continue comparing the colors in the two windows alternately. In this case, the complementary after-image of each color is piled up on the other color. Even a slight color difference is then emphasized. In other words, if it is quite the same color, it does not look anything else than quite the same color. Therefore, this method is very handy and accurate if we become used to it. However, the phenomenon of a color difference being emphasized when two neighboring colors are compared differs entirely in meaning from the phenomenon of quite the same color looking quite the same color.

\section{Color-Mixing and Color-Matching}

Color-mixing is divided roughly into additive color mixture and subtractive color mixture, with mean color mixture included in special cases. The third should properly be inclined in additive color mixture, but it had better be separated ostensibly.

For the sake of convenience, I illustrate subtractive color mixture at first. I used printing ink because, I had to make a basic experiment requiring the use of colored materials of a high degree of transparence. The purpose of my basic experiment was to decide whether it was right to use printing ink, because I had noticed the difficulty of making color votes with printing ink when, in 1942, I co-operated in compiling the Dictionary of Color Names in Japan by Dr. Rokuro Uemura, famous for his study in the culture of dyeing, at his request.

Later, I made a series of experiments in an effort to produce, with printing ink, the same colors as color samples and discovered, from among inks of more than 10 dozen different colors, those satisfactory and adequate for my purpose. (I acknowledge here the co-operation of Morohashi Chemical Industry Co., Yokohama, in my discovery.) The inks I discovered were:

$\begin{array}{lll}\text { Red } & 2.5 \mathrm{R} 4 / 14 & 1 \\ \text { Orange red } & 7.5 \mathrm{R} 5 / 14 & 2 \\ \text { Yellow } & 5 \mathrm{Y} 8 / 12 & 3 \\ \text { Green } & 2.5 \mathrm{G} 5 / 10 & 4 \\ \text { Greenish blue } & 7.5 \mathrm{~B} 4 / 8 & 5 \\ \text { Purplish blue } & 7.5 \mathrm{~PB} 3 / 12 & 6 \\ \text { Red purple } & 2.5 \mathrm{RP} 4 / 14 & 7 \\ \text { White } & \mathrm{N} 9.5 / 0 & 8 \\ \text { Black } & \mathrm{N} 1.5 / 0 & 9\end{array}$

Note: Revised Mansell notations are based on standard color votes.

I made repeated experiments in color-mixing by using white, black and the other seven inks listed above. I became able freely to produce, by color-mixing, the colors of dyeing and weaving handed down from ancient times in Japan.

Let me explain briefly, with the record of my experiments, color-matching by color-mixing. Dilute primary ink with white ink until light scatters and a bluish tint is added to the primary ink. In other words, dilution with white ink changes the hue of the primary ink. The change needs correction. For example, $2.5 \mathrm{R}$, diluted, turns, successively, bluish rose and bluish pink. To erase this bluishness, mix a modicum of $7.5 \mathrm{R}$ with $5 \mathrm{Y}$ and add a modicum of yellow orange obtained from the mixture to $2.5 \mathrm{R}$. We then obtain a rose or pink which belongs to the $2.5 \mathrm{R}$ group.

This procedure is highly useful for making grey. The usual method of making grey of N6.5/0 is to add a small amount of black to white and obtain grey similar to N6.5/0 in the degree of brightness, but the grey thus obtained is bluish grey. Then add the yellow orange obtainable from the mixture of $7.5 \mathrm{R}$ and $5 \mathrm{Y}$, just mentioned, until the bluishness vanishes. The grey finally obtained is hardly distinguishable from the standard color vote of $\mathrm{N} 6.5 / 0$. 
Now to explain how to produce various colors of greyish tone by mixing white with three primary colors - red, yellow and blue - without using any black at all. In this case, we have to do color-matching by visualizing a color triangle and judging the inclination of the hue by the sense of vision. Therefore, only experience of color-matching does not help.

The three primary colors are $2.5 \mathrm{R}, 5 \mathrm{Y}$ and $7.5 \mathrm{~B}$, but, in some cases, we may have to use additionally $R$. $5 \mathrm{R}$ and $2.5 \mathrm{G}$ or $7.5 \mathrm{~PB}$ and $2.5 \mathrm{RP}$. Usually, however, the first three are sufficient. Proper mixing of the trio gives us an almost black, but it is noticeably different from N1.5/0 . Here, however, we need not obtain pure black. All we have to do is to notice and remember the ratios of the three colors necessary for mixing to obtain a near-black.

Now take a beige of color sample and try to make a matching color. Begin by adding red and yellow to white until the mixture is a yellow orange. Then add a small amount of blue gradually until the reddishness disappears.

This does not mean that exact color-matching is possible after one or two attempts, but it does show that by the procedure just described we can get close to beige. That is all we have to know. The rest that we have to do is to be careful of the sensibility of our eyes and pay attention to the eye measurement of the amount of ink.

To make a reddish dark color comparative low in chroma, such as maroon, add green to red, lower their value and chroma and increase the value of white. This gives us the average maroon. If fashion demands a purplish or yellowish maroon, we can make a purplish maroon by adding a small amount of $2.5 \mathrm{RP}$, or a yellowish maroon by adding a small quantity of $7.5 \mathrm{R}$.

A brown color like that of tobacco can be produced by adding $7.5 \mathrm{~B}$ to $7.5 \mathrm{R}$ and mixing them with white and $5 \mathrm{Y}$. Moss green can be obtained by adding a small quantity of $7.5 \mathrm{R}$ to $2.5 \mathrm{G}$ or $5 \mathrm{Y}$ to $7.5 \mathrm{~PB}$.

These procedures merely apply the theory of the relations between complementary colors. Actually, we must measure changes in the shade of each color with a recording spectro photometer and obtain spectral reflectance curves.

\section{Multi-Color Effect of Two-Color Printing}

In 1946 I accepted an invitation to become an adviser to Yamamoto Ink Co., Ltd., Osaka, and started research into reinforcing the blackness of black ink newspaper printing at the request of Ieo Yamamoto, president. Carbonblack available then was made in Japan, of poor quality and inadequate blackness. Besides, the whiteness of newsprint was so poor that the blackand-white contrast made reading extremely difficult.

The first task for the Yamamoto firm was to im. prove the blackness of ink, and the task devolved on me. I was happy that the task entailed an additional task: research into the multi-color effect of two-color printing. I began by making two groups of negatives, one for three-color printing and one for two-color printing, from negatives for photo-color printing and studied color print ing. Finally $I$ was able to make the effects of twoand three-color printing closely resemble each other. The two are difficult to be distinguished if separately observed.

Marvelous work was done in making the plates for my researches by Mizuo Nagata, president of Nagata Printing Co., Osaka. Not only his technique in platemaking and his sharpness of empirical color sensibility born of many years' experience, but also his zeal for the revolutionary researches brought them to fruition. The following table, it is hoped, will give an idea of the researches and their results:

Inks used Orange red (OR) $10 \mathrm{R} 6 / 14$

Blue green (BG) $7.5 \mathrm{G} 6 / 8$

Color by net point Effect of complementary contrast

a) OR (Orange red)

b) BG (Blue green)

Pale blue green

c) Paper (White)

d) $\mathbf{a}-\mathbf{b}$ (Black) (subtraction)

Pink $_{1}$

e) $a+b$ (Yellow) (Fusion)

Dark tone

f) $a+c$ (Rose - Pink) (Fusion)

Bright tone

g) $\mathbf{b}+\mathbf{c}$ (Pale blue green) (Fusion) Pink $_{2}$

h) $\mathrm{a}+\mathrm{d}$ (Brown) (Fusion) Pale Blue

i) $\mathbf{b}+\mathbf{d}$ (Dark blue green) (Fusion) Pink $_{3}$

The number of colors given out by combination of net points

......... n

Gradations of shade by variations of the size of net point

......... 10

The number of changes of the ground color and pattern

$\ldots \ldots \ldots 2$

Total of color effects......... S

$$
S=10 n+2 n(n-1)=234
$$

\section{Conclusions}

It is difficult to explore the unknown world of color with the explorer doing trail-blazing himself. It is still more difficult to win understanding of the results of exploration and co-operation in further exploration. This I have been saying for 40 years. 
Yet I have the satisfaction of knowing that my 40 years' labors in the exploration of the world of color have been rewarding. I have received expressions of appreciation from the Kansai Electric Power Co. for the color control I introduced into their Generation Station No. 4 at Kurobe, Toyama Prefecture; from hosts of dyers, weavers, apparel makers and beauticians for my guidance and advice. To the latter group I owe a debt of gratitude for their co-operation in my tests of character by color effect.

This article touches a mere fringe of the subject or color, but I am glad to have had an opportunity of publishing it to the world. Grateful appreciation is due to The Textile Machinery Society of Japan for giving me that opportunity. I wish the society steady future growth. 